\title{
ADOLESCENTE CON INESTABILIDAD LUMBOSACRA POR PRESENCIA DE QUISTE HIDATÍDICO: REPORTE DE CASO
}

\author{
Miguel Vizcarra1,a,b, Mijaíl Mujica ${ }^{1, a, b}$, Vladimir Sánchez ${ }^{1, a, b}$, Paul Méndez
}

\begin{abstract}
RESUMEN
La hidatidosis, causada por el Echinococcus granulosus, afecta a la columna vertebral en $0,5-2 \%$ del total de casos, siendo la columna sacra una ubicación rara, típica de personas en edad adulta. Se reporta el caso de un varón de 14 años oriundo de Juliaca (Puno, Perú) quien presentó lumbalgia crónica y síndrome de cauda equina. Atendido inicialmente en Juliaca, fue trasladado al Hospital Edgardo Rebagliati Martins (Lima, Perú) con el diagnóstico de tumoración sacra. En la resonancia magnética se mostró una tumoración multiquística con lisis del L5 y S1 e inestabilidad. El tratamiento quirúrgico fue una laminectomía L5-S2 y extracción de múltiples quistes de hidátide más estabilización lumbopélvica con tornillos transpediculares. En el posoperatorio se recetó albendazol $(15 \mathrm{mg} / \mathrm{kg} / \mathrm{d})$ por el periodo de seis meses. Se debe considerar al quiste hidatídico dentro del diagnóstico diferencial cuando existan lesiones quísticas en la columna vertebral, sobre todo en regiones endémicas como el Perú.
\end{abstract}

Palabras clave: Equinococosis; Región lumbosacra;/cirugía (fuente: DeCS BIREME).

\section{AN ADOLESCENT WITH LUMBOSACRAL INSTABILITY DUE TO THE PRESENCE OF A HYDATID CYST: A CASE REPORT}

\begin{abstract}
Hydatidosis, caused by Echinococcus granulosus, affects the spinal column in $0.5-2 \%$ of all cases, with the sacral column being a rare location typically observed only in adults. The case is reported of a 14-year-old boy from Juliaca (Puno, Peru) who presented with chronic lower back pain and cauda equina syndrome. He was initially attended to in Juliaca, and then transferred to the Hospital Edgardo Rebagliati Martins (Lima, Peru) with a diagnosis of sacral tumor. In magnetic resonance imaging, a multicystic tumor was seen with L5 and S1 lysis and instability. The surgical treatment was an L5$\mathrm{S} 2$ laminectomy and extraction of multiple hydatid cysts followed by lumbopelvic stabilization with transpedicular screws. Post-operatively, he was prescribed albendazol $(15 \mathrm{mg} / \mathrm{kg} / \mathrm{d})$ for six months. Hydatid cysts should be considered within the differential diagnosis when there are cystic lesions in the spinal column, particularly in endemic areas such as Peru.
\end{abstract}

Key words: Echinococcosis; Lumbosacral region; /surgery (source: MeSH NLM).

\section{INTRODUCCIÓN}

La hidatidosis es una infestación zoonótica causada por el Echinococcus granulosus que, frecuentemente, ocurre en lugares donde conviven el hombre, un rumiante portador de larva (principalmente ovino) y un carnívoro (principalmente perros) portador del cestodo adulto. Los huevos del parásito se encuentran en las heces de los caninos y son la fuente de infestación de los huéspedes intermediarios (hombre) en los cuales se desarrolla el quiste hidatídico (1).
La hidatidosis tiene distribución mundial y en Sudamérica es endémica en países como Uruguay, Argentina, Brasil, Perú, Bolivia y Chile (2). En el Perú, entre los años 20012006, se presentaron entre 7 y 11 casos de hidatidosis por cada 100000 habitantes; entre los departamentos con mayor incidencia se encuentran: Junín, Cerro de Pasco y Huancavelica ${ }^{(3)}$.

El costo ocasionado por la hidatidosis involucra costos médicos (atención médica, cirugía si requiriera, estancia hospitalaria, medicación), y no médicos (días

\footnotetext{
Departamento de Neurocirugía. Hospital Nacional Edgardo Rebagliati Martins. Lima, Perú.

Universidad Nacional de Trujillo. Trujillo, Perú.

Médico cirujano; bespecialista en Neurocirugía.

Recibido: 04/08/2016 Aprobado: 08/02/2017 En línea: 23/03/2017
}

Citar como: Vizcarra M, Mujica M, Sánchez V, Méndez P. Adolescente con inestabilidad lumbosacra por presencia de quiste hidatídico: reporte de caso. Rev Peru Med Exp Salud Publica. 2017;34(1):126-31. doi: 10.17843/rpmesp.2017.341.2775 
no trabajados, ausentismo escolar, consecuencias emocionales, sociales y económicas). Un estudio realizado en el Instituto Nacional del Niño del Perú, para el periodo 2006-2010, concluyó que el promedio de estancia hospitalaria fue de 34 días, el costo por paciente con tratamiento quirúrgico fue de 914,3 dólares, en aquellos en los que no se realizó cirugía fue de 298,7 dólares por persona ${ }^{(5)}$. En otro estudio se estimó que el costo total (costo del tratamiento más costo por pérdida de productividad) que origina la hidatidosis en el Perú fue de 2420348 dólares por año ${ }^{(6)}$.

La infestación de hidatidosis se ubica, principalmente, en el pulmón y en el hígado ${ }^{(4)}$. La hidatidosis vertebral ocurre entre el 0,5 y $2 \%$ de todos los casos y corresponde al $50 \%$ de todas las hidatidosis musculoesqueléticas; es seguida, en frecuencia, por otras localizaciones como la pelvis y huesos largos ${ }^{(7,12)}$. Estas lesiones se localizan en la región dorsal $(52 \%)$, lumbar $(37 \%)$ y cervical - sacra $(11 \%)^{(7,8)}$, siendo la columna sacra una ubicación rara ${ }^{(8,9)}$.

Presentamos el caso de un paciente con quiste hidatídico localizado en región lumbosacra, cuya manifestación clínica fue el síndrome de cauda equina e inestabilidad lumbosacra. El paciente dio su consentimiento para reportar el caso.

\section{REPORTE DEL CASO}

Paciente varón de 14 años procedente de la cuidad de Juliaca, región Puno - Perú, sin antecedentes de importancia, quien relata que presentó dolor de tipo punzante en la zona lumbosacra, de moderada intensidad que fue progresando durante los dos últimos años tras sufrir una caída desde una altura aproximada de 1,5 m. Durante el último año, se agregó la pérdida progresiva de sensibilidad en ambos miembros inferiores a predominio distal. Un mes antes de la consulta, presentó pérdida progresiva de fuerza en ambos miembros inferiores a predominio distal, hasta solo movilizarse en silla de ruedas al final del periodo.

Acudió a establecimiento de salud de nivel primario y luego fue derivado a Hospital III base Salcedo de la ciudad de Puno, región Puno - Perú en donde, al examen clínico, se encontró: lumbalgia moderada de tipo punzante, paraparesia 3/5 e hipoestesia +/+++ en miembros inferiores. El estudio tomográfico mostró una tumoración que erosionaba la última vértebra lumbar (L5) y el hueso sacro (Figura 1). Posteriormente,
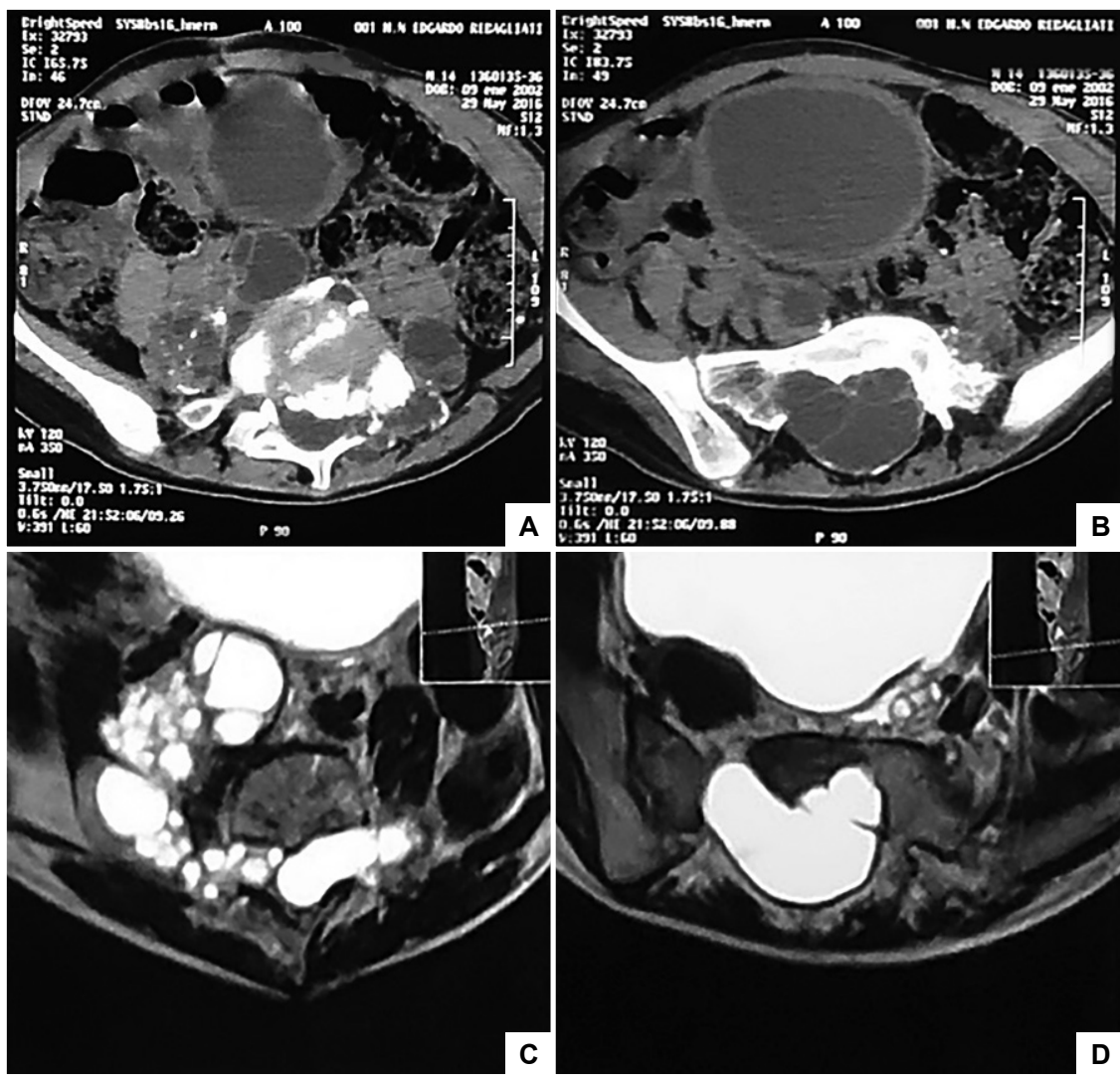

Figura 1. A y B. Tomografías axiales sin contraste en donde se evidencia una tumoración quística que erosiona el cuerpo vertebral en L5 y S1 e invade la región abdominal. C y D. Resonancia magnética en T2 donde también se muestra tumoración quística que invade el canal espinal en L5 y S1. 
fue referido al Hospital Edgardo Rebagliati Martins (HNERM) de la ciudad de Lima, Perú. Al ingreso a la emergencia, no hubo signos resaltantes en la ectoscopía ni en el examen físico general; al examen neurológico: paciente despierto, orientado, con paraparesia 2/5 a predominio distal, sensibilidad $+/+++$ en silla de montar, reflejos osteotendinosos $+/+++$; reflejo cutáneo plantar indiferente bilateral, y ausencia de control de esfínteres. Se aprecia hipotrofia muscular bilateral a predominio de miembro inferior izquierdo ++/+++.

En los exámenes complementarios, el hemograma, el perfil de coagulación, el perfil bioquímico y los electrolitos se encontraban dentro de los rangos normales.

En la evaluación por resonancia magnética (RMN) lumbosacra se identificó: en secuencia T1, tumoración mixta (sólido-quística) que compromete $2 / 3$ del hueso sacro y todo el cuerpo de L5, e invade canal raquídeo; en T2 se aprecia que dicha tumoración ocasiona laterolistesis L5-S1 y compresión de la cauda equina en dicho nivel (Figura 1); en T1 con contraste, se aprecia escasa captación de contraste en dicha masa (Figura 2).

En la tomografía lumbosacra con reconstrucción 3D, se aprecia una tumoración de forma irregular que produce lisis de la vértebra L5 y el hueso sacro, laterolistesisL5-S1 hacia la derecha e inestabilidad lumbosacra debido a la rotura del arco posterior de L5 y S1 (Figura 3). Con dichos hallazgos se decidió realizar la intervención quirúrgica.

Se realizó una laminectomía L5-S2. Se extrajeron múltiples quistes hidatídicos compuestos por membranas delgadas, de contenido claro-amarillento, que se encontraban impregnados en el hueso sacro y el saco dural; antes de la extracción de los quistes se irrigó la zona con solución salina hipertónica. Luego, se realizó la estabilización lumbopélvica mediante la técnica de Galveston modificada; se realizó la fijación transpedicular (FTP) lumbar en vertebras L3, L4 y L5 con seis tornillos de $5,5 \times 40 \mathrm{~mm}$, y fijación a los huesos iliacos con cuatro tornillos -dos $5,5 \times 55 \mathrm{~mm}$ y dos de 6,0 × $60 \mathrm{~mm}$ - (Figura 4 y 5). No hubo complicaciones durante la cirugía. El estudio anatomopatológico reveló quiste hidatídico no viable (Figura 4).

En el posquirúrgico, se indicó el uso de albendazol $15 \mathrm{mg} / \mathrm{kg} / \mathrm{d}$ durante 6 meses. Al cuarto día posquirúrgico se realizó una tomografía lumbosacra con reconstrucción 3D en donde se evidenciaba estabilización lumbopélvica, corrección de la laterolistesis y reconstrucción de la anatomía espinal (Figura 5). El paciente refirió mejoría en el control de esfínteres; al décimo día posquirúrgico presentó mejoría de la fuerza muscular en ambos miembros inferiores.
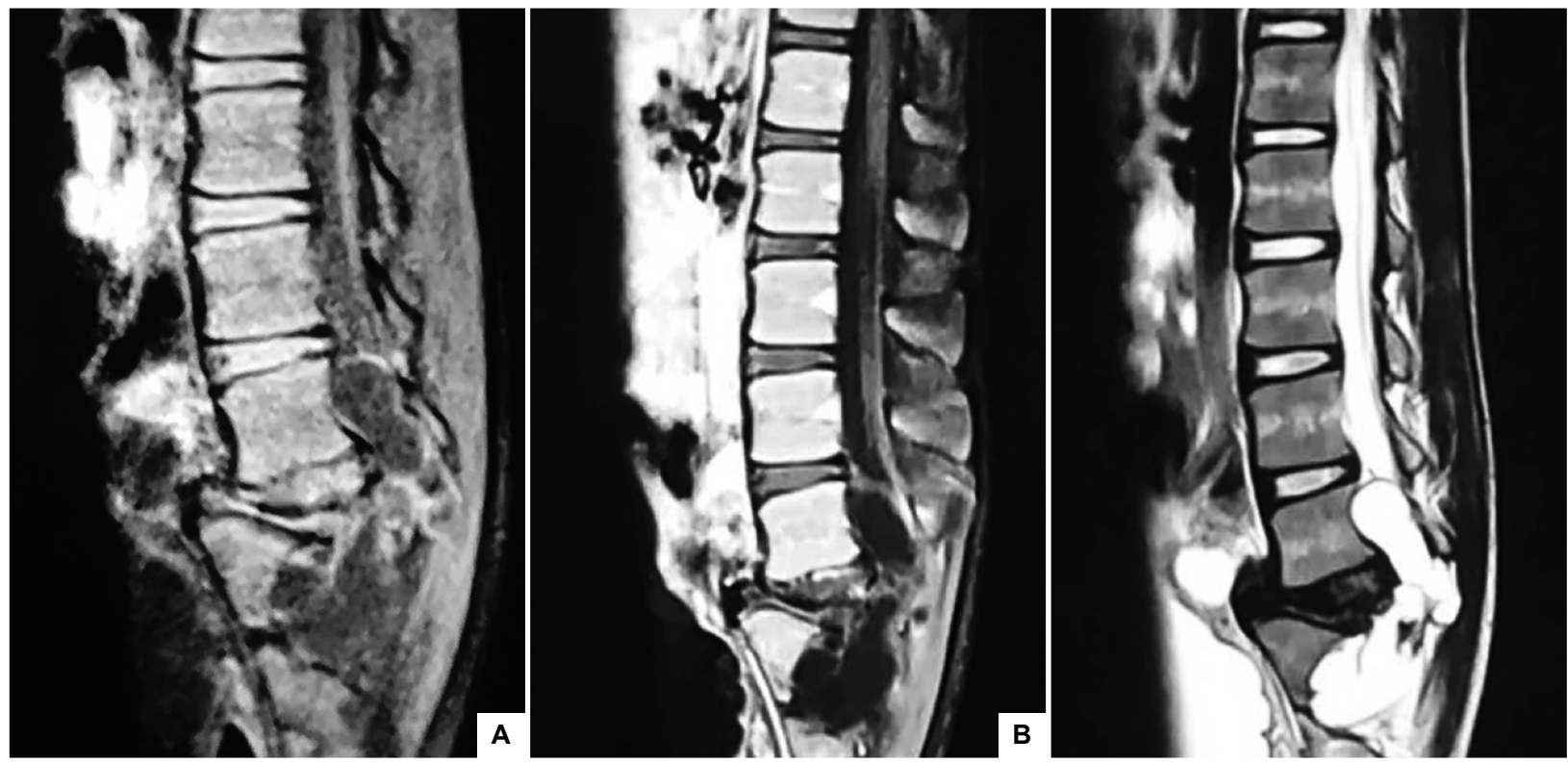

Figura 2. Resonancia magnética (RM), cortes sagitales. A. RM en T1 con contraste que muestra tumoración lumbosacra con escasa captación de contraste. B. RM en T1 sin contraste que muestra tumoración hipointensa y lisis de las vértebras L5 y S1. C. RM en T2 se aprecia tumoración hipointensa, signo de su contenido quístico; además, listesis lumbosacra y la invasión del canal espinal. 

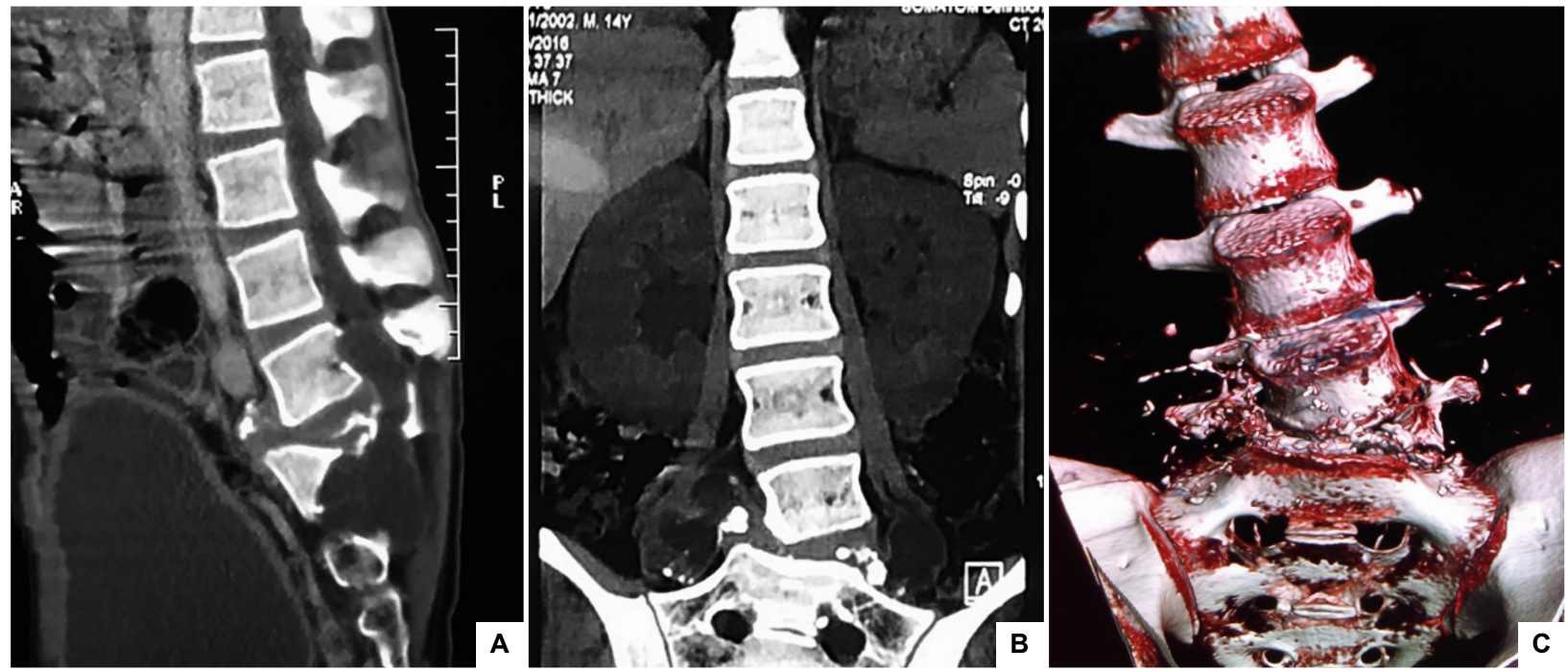

Figura 3. Tomografías sin contraste, cortes sagital (A) y coronal (B) en los que se observa una tumoración quística que erosiona cuerpos vertebrales y genera una laterolistesis en L5 y S1. C. Reconstrucción en 3D de columna vertebral en el que se aprecia la erosión ocasionada y la inestabilidad lumbosacra.

\section{DISCUSIÓN}

La hidatidosis ósea es una enfermedad poco común, aunque aumenta su frecuencia en países endémicos como el Perú $(4,5)$; nuestro paciente provenía de la región Puno, que es una zona ganadera. La hidatidosis sacra es una entidad rara ${ }^{(5,7)}$; así, en un estudio de 25 casos de hidatidosis espinal solo se encontraron seis casos que afectaban al hueso sacro ${ }^{(8)}$, es por ello que nuestro caso es interesante y nos llevó a plantear diagnósticos diferenciales. Aun más raro fue que presentó inestabilidad lumbosacra ocasionada por la laterolistesis. La ubicación de este tipo de hidatidosis es extradural en el $90 \%$ de los casos, e invade cuerpo vertebral ${ }^{(10)}$ y coincidió con nuestro caso.

La hidatidosis espinal es más frecuente en personas adultas (11), mientras que en nuestro paciente la sintomatología se inició a los 14 años. Las manifestaciones clínicas no son específicas y son consecuencia de la comprensión del cordón espinal. Estas manifestaciones pueden presentarse como
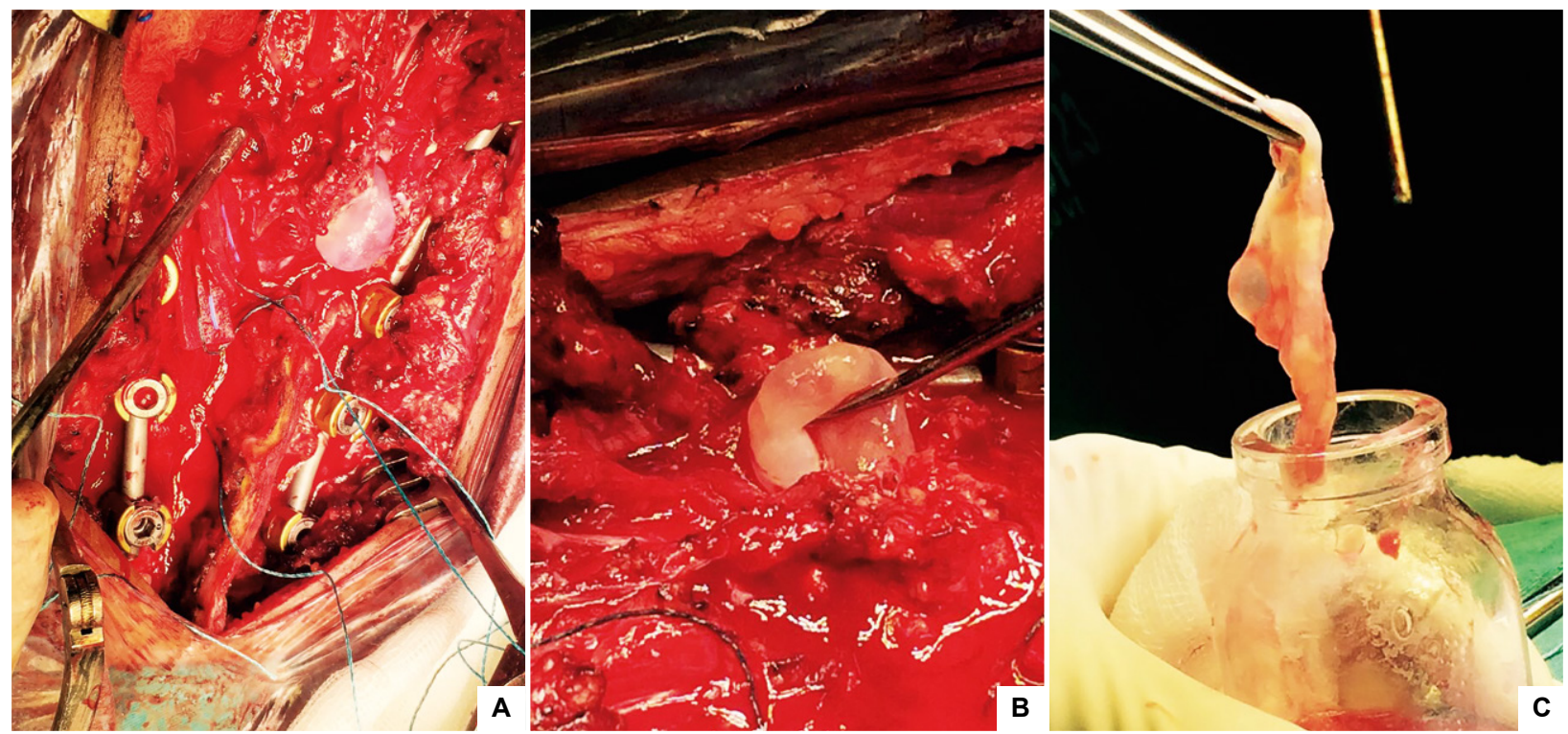

Figura 4. Imágenes intraoperatorias. A y B. Se observa la presencia de quistes distribuidos en la zona sacra. C. Tejido quístico recuperado para evaluación anatomopatológica. 

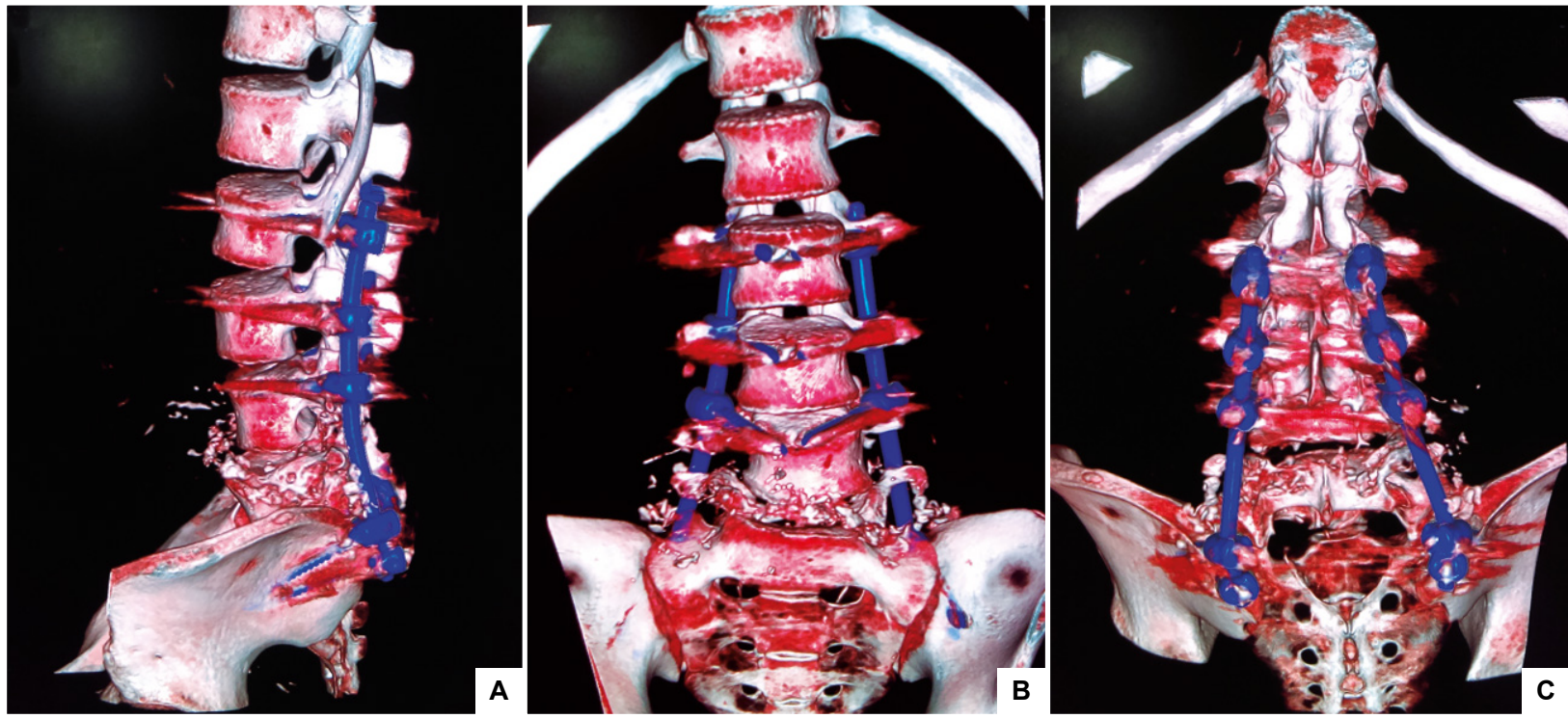

Figura 5. Reconstrucciones en 3D de columna vertebral posquirúrgicas. Corte sagital (A) y coronal - anteroposterior (B) y posteroanterior (C)-. Se muestra el resultado de la reconstrucción de la anatomía (corrección de la laterolistesis) y estabilización lumbosacra con la utilización del sistema de fijación transpedicular, de color azul.

dolor lumbar (85\%), dolor radiculopático (25-95\%), paraparesia $(25-77 \%)$ y retención urinaria en pocos casos ${ }^{(12)}$. Nuestro paciente presentó dolor lumbar seguido de compromiso neurológico.

Por los estudios de imágenes podemos sospechar la presencia de un quiste hidatídico espinal cuando evidenciamos la presencia de una lesión lítica con borde esclerosado; dicha lesión es de aspecto quístico multilocular (un quiste precursor y quistes "hijos"), y no afecta el espacio discal; en RMN en T1 el quiste multilocular tiene una intensidad de señal similar al músculo, en T2 los quistes hijos y el precursor son hiperintensos, en T1 con contraste de gadolinio no hay captación del contraste ${ }^{(9,15)}$. Dentro de los diagnósticos diferenciales se encuentran quiste dermoide, meningocele sacro, schwanoma y quiste aracnoideo, entre otros ${ }^{(9,10)}$. En nuestro caso, identificamos una imagen multiquística hiperintensa en T2, así como lisis de la región posterior del sacro y cuerpo vertebral de L5. Dentro de la clasificación de Braitwate and Lees, correspondería a un quiste hidatídico paravertebral e intraespinal (tipo 3 y 5$)^{(10)}$.

El tratamiento implica el abordaje quirúrgico y farmacológico, siendo este último administrado en el posoperatorio para controlar la enfermedad, evitar la diseminación sistémica y posible recurrencia ${ }^{(12)}$. El tratamiento quirúrgico consta de la descompresión (laminectomía y/o facetectomía), exéresis de los quistes, liberación del canal espinal y estabilización (fijación transpedicular) ${ }^{(8,10,15)}$. A pesar del tratamiento quirúrgico, la enfermedad con frecuencia recae con destrucción progresiva de la columna vertebral y deterioro neurológico ${ }^{(15)}$. En nuestro caso, se realizó un abordaje posterior, laminectomía y extracción de múltiples quiste con irrigación de solución hipertónica con cloruro de sodio al $20 \%$.

Algunos pacientes pueden requerir estabilización espinal, dependiendo si existen signos de inestabilidad por rotura de arcos posterior del cuerpo vertebral o destrucción de pedículos o si hubo descompresión amplia; cuando la estabilización es con tornillos pediculares, el cirujano debe ser consciente de que la enfermedad podría diseminarse en los cuerpos vertebrales si existen escólex viables, por lo que deben revisarse los pedículos antes de la inserción de tornillos ${ }^{(13,14)}$. Nuestro paciente tenía signos de inestabilidad y laterolistesis lumbosacra, por lo cual se realizó la estabilización lumbopélvica. Esta característica no se ha reportado en las publicaciones revisadas.

Podemos concluir que debe considerarse al quiste hidatídico espinal dentro del diagnóstico diferencial cuando existen lesiones quísticas que originan osteolisis y compresión medular; además, debe valorarse si se requiere la estabilización espinal mediante cirugía, todo ello con la finalidad de brindar al paciente un tratamiento óptimo y una pronta recuperación, y así rehabilitarlo para sus actividades diarias y laborales.

Contribuciones de autoría: MV, MM, VS y PM participaron en la concepción del artículo, la recolección de datos, redacción y aprobación de la versión final del artículo. PM realizó revisión bibliográfica.

Fuentes de financiamiento: autofinanciado.

Conflicto de intereses: los autores declaran no tener ningún conflicto de interés. 


\section{REFERENCIAS BIBLIOGRÁFICAS}

1. Martínez P. Hidatidosis humana en menores de edad: manifestación de fracaso en las medidas de control y prevención. Chile, 2001-2011. Rev Chilena Infectol. 2015;32(2):158$66 . \quad$ doi: $10.4067 /$ S071610182015000300004

2. Cucher M, Macchiaroli N, Baldi G, Camicia F, Prada L, Maldonado $\mathrm{L}$, et al. Cystic echinococcosis in South America: systematic review of species and genotypes of Echinococcus granulosus sensu lato in humans and natural domestic hosts. Trop Med Int Health. 2016;21(2):166-75. doi: $10.1111 /$ tmi. 12647

3. Salgado $S$, Suarez-Ognio L, Cabrera R. Características clínicas y epidemiológicas de la equinococosis quística registradas en un área endémica en los andes centrales del Perú (1991-2002). Neotrop Helminthol. 2007;1(2):69-83.

4. Montúfar-Valer A, HuapayaJurado FL. Características clínicas, radiológicas y laboratoriales de pacientes con hidatidosis hepática en un hospital de referencia nacional, Lima 1997-2010. Rev Gastroenterol Peru. 2014;34(3):203-9

5. Fano $\mathrm{P}$, Hernández $\mathrm{H}$ y Falcón $\mathrm{N}$. Costos de la atención de pacientes con equinococosis quística humana en un hospital de niños en Lima-Perú, periodo 2006-2010. Salud tecnol. vet. 2014;2(2): 63-70. doi: 10.20453/stv. v2i2.2248

6. Moro P, Budke C, Schantz P, Vasquez J, Santivañez S, Villavicencio J. Economic impact of cystic echinococcosis in Peru. PLoS Negl Trop Dis. 2011;5(5):e1179. doi: 10.1371/journal.pntd.0001179

7. Neumayr A, Tamarozzi F, Goblirsch S, Blum J, Brunetti E. Spinal cystic echinococcosis - a systematic analysis and review of the literature: part 1 . Epidemiology and anatomy. PLoS Negl Trop Dis. 2013;7(9):e2450. doi: 10.1371/journal.pntd.0002450

8. Abdelhakim K, Khalil A, Haroune B, Oubaid M, Mondher M. A case of sacral hydatid cyst. Int J Surg Case Rep. 2014;5(7):434-6. doi: 10.1016/j. ijscr.2014.03.025

9. Song $\mathrm{X}$, Liu $\mathrm{D}$, Wen $\mathrm{H}$. Diagnostic pitfalls of spinal echinococcosis. J Spinal Disord Tech. 2007;20(2):180-5. doi: 10.1097/01. bsd.0000211288.37013.42

10. Neumayr A, Tamarozzi F, Goblirsch S, Blum J, Brunetti E. Spinal cystic echinococcosis - a systematic analysis and review of the literature: part 2 . Treatment, follow-up and outcome.
PLoS Negl Trop Dis 2013;7(9):e2458. doi: 10.1371/journal.pntd.000245

11. Hamdan T. Hydatid disease of the spine: a report on nine patients. Int Orthop. 2012;36(2):427-32.

12. Hemama M, Lasseini A, Rifi L, Boutarbouch M, Derraz S, Ouahabi AE, et al. A sacral hydatid cyst mimicking an anterior sacral meningocele. J Neurosurg Pediatr. 2011;8(5):526-9. doi: 10.3171/2011.8.PEDS10555

13. Khazim RM: Spinal hydatid disease. South Med J. 2006;99(2):114. doi: 10.1097/01.smj.0000198264.01011. d 1

14. Patel D, Shukla D. Back bugged: A case of sacral hydatid cyst. J Neurosci Rural Pract. 2010;1(1):43-5. doi: 10.4103/0976-3147.63104

15. Senoglu M, Bulbuloglu E, Demirpolat G, Altun I, Celik M. Combined anterior and posterior approach for sacral/retroperitoneal hydatid cyst disease: case report. Turk Neurosurg. 2009;19(4):428-32.

Correspondencia: Paul Martín Méndez Aguilar Dirección: Jr. Mariscal Miller 1840

Teléfono: (+51) 949180420

Correo electrónico:pmma85@gmail.com

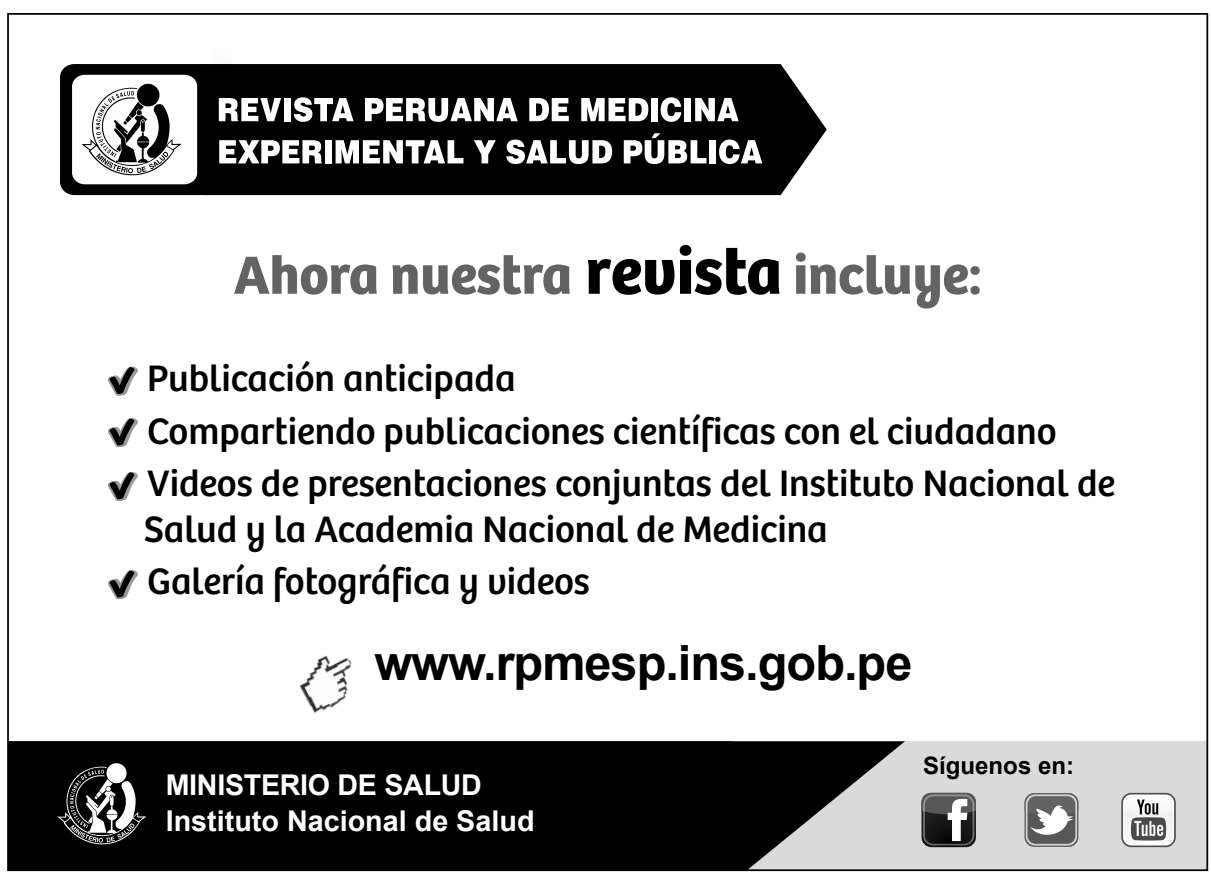

